INPLASY

PROTOCOL

To cite: Cao et al. The relationship between obstructive sleep apnea hypopnea syndrome and interstitial lung diseases: an uptated meta-analysis. Inplasy protocol 202170085. doi: 10.37766/inplasy2021.7.0085

Received: 26 July 2021

Published: 26 July 2021

Corresponding author: Yanmei Cao

cymei1227@163.com

Author Affiliation:

The Second Affiliated Hospital of Soochow University.

Support: SZYJTD201904.

Review Stage at time of this submission: Data analysis.

\section{The relationship between obstructive sleep apnea hypopnea syndrome and interstitial lung diseases: an uptated meta-analysis}

Cao, YM¹; Xie, LB2; Wang, P3; Chen $\mathrm{R}^{4}$.

Review question / Objective: A meta-analysis was performed to identify the association between obstructive sleep apnea and interstitial lung diseases.

Condition being studied: The relationship between obstructive sleep apnea hypopnea syndrome and interstitial lung diseases. Four databases including EMBSAE, PubMed, Web of Science and Cochrane Library were searched up to March 2021. The data extraction has been completed.

INPLASY registration number: This protocol was registered with the International Platform of Registered Systematic Review and Meta-Analysis Protocols (INPLASY) on 26 July 2021 and was last updated on 26 July 2021 (registration number INPLASY202170085).

Conflicts of interest:

None declared.

\section{INTRODUCTION}

Review question / Objective: A metaanalysis was performed to identify the association between obstructive sleep apnea and interstitial lung diseases.

Condition being studied: The relationship between obstructive sleep apnea hypopnea syndrome and interstitial lung diseases. Four databases including EMBSAE,
PubMed, Web of Science and Cochrane Library were searched up to March 2021. The data extraction has been completed.

\section{METHODS}

Search strategy: The search terms were [(Sleep Apnea Syndromes) OR (Snoring) OR ((sleep* and (apnea* or apnoea* or hypopnea* or hypopnoea*)) OR (sleep* and disorder* and breath*) OR (OSA or OSAS or 
OSAHS or SAHS)] AND [(Lung Diseases, Interstitial) OR ((pulmonary* or lung*) and (fibros* or fibrot*)) OR (sarcoidosis* or alveolitis*) OR(ILD)].

Participant or population: Patients who were diagnosed interstitial lung diseases.

Intervention: None.

Comparator: None.

Study designs to be included: Observational studies.

Eligibility criteria: The patients who were dianosed interstitial lung diseases.

Information sources: The databases that were searched included EMBSAE, PubMed, Web of Science and Cochrane Library.

Main outcome(s): The pool prevalence of obstructive sleep apnea in interstitial lung diseases patients.

Quality assessment / Risk of bias analysis: The NewcastleJCOttawa scale (NOS) was selected to assess the quality of casecontrol or cohort studies. The Agency for Healthcare Research and Quality (AHRQ) checklist was chosen to assess the quality of cross-sectional studies.

Strategy of data synthesis: Statistic Analyses were performed using the STATA 16.

Subgroup analysis: Subgroup analyses by the different diseases of ILD and the method for OSA.

Sensitivity analysis: After sequentially excluding each study, we failed to find any study that could have an effect on the final outcome.

Country(ies) involved: China.

Keywords: OSA; Obstructive Sleep Apnea; ILD; Interstitial Lung Diseases; Metaanalysis.
Contributions of each author:

Author 1 - Yanmei Cao.

Author 2 - Liangbin Xie.

Author 3 - Ping Wang.

Author 4 - Rui Chen. 